Document downloaded from:

http://hdl.handle.net/10251/103259

This paper must be cited as:

Sanz Diaz, R.; García Gil, PJ.; Albertos Pérez, P. (2017). A generalized smith predictor for unstable time-delay SISO systems. ISA Transactions. 72:197-204.

doi:10.1016/j.isatra.2017.09.020

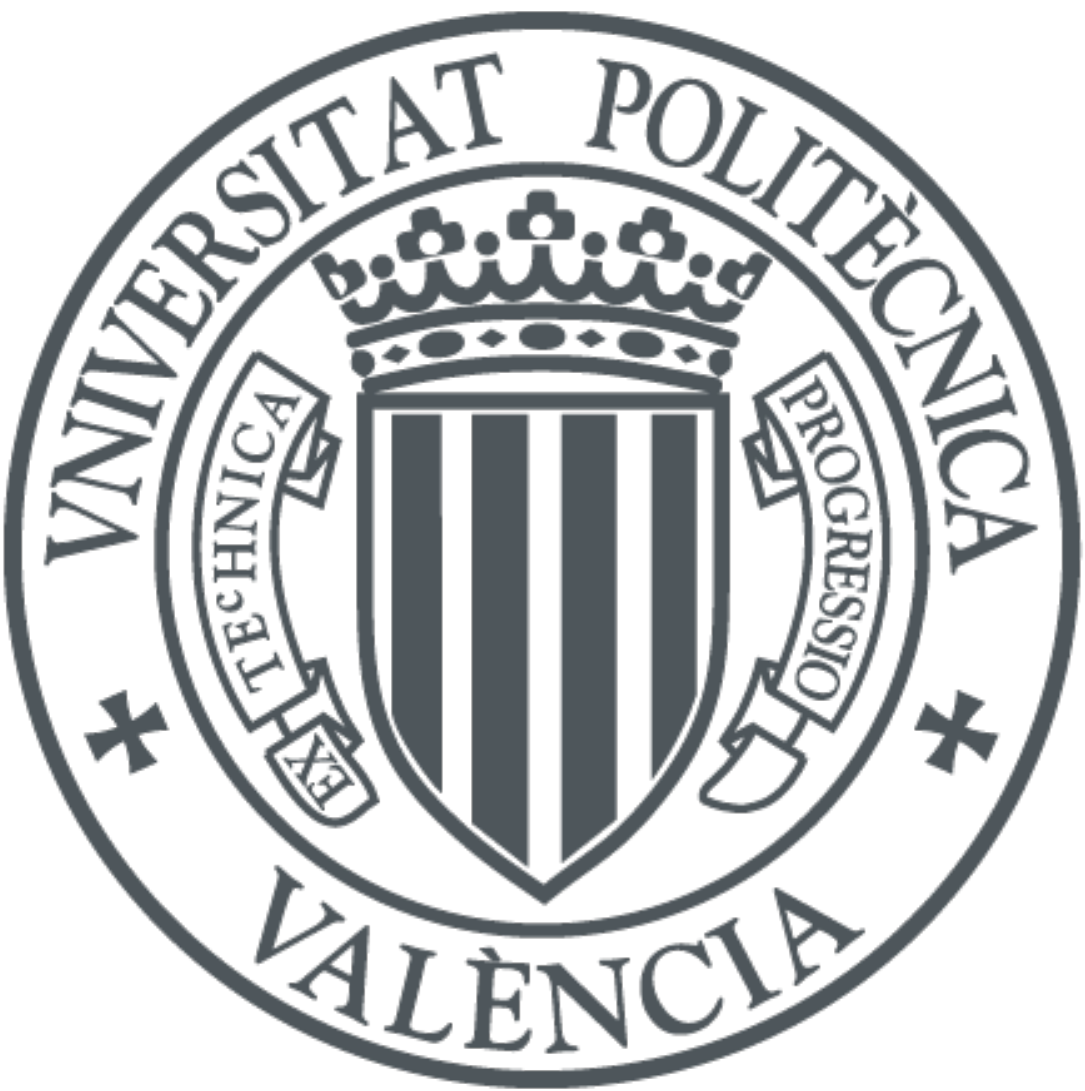

The final publication is available at

https://doi.org/10.1016/j.isatra.2017.09.020

Copyright Elsevier

Additional Information 


\title{
A Generalized Smith Predictor for Unstable Time-Delay SISO Systems
}

\author{
R. Sanz ${ }^{\mathrm{a}, *}$, P. García ${ }^{\mathrm{a}}$, P. Albertos ${ }^{\mathrm{a}}$ \\ ${ }^{a}$ Instituto de Automática e Informática Industrial \\ Universitat Politècnica de València, 46020 València, Spain
}

\begin{abstract}
In this work, a generalization of the Smith Predictor (SP) is proposed to control linear time-invariant (LTI) time-delay singleinput single-output (SISO) systems. Similarly to the SP, the combination of any stabilizing output-feedback controller for the delay-free system with the proposed predictor leads to a stabilizing controller for the delayed system. Furthermore, the tracking performance and the steady-state disturbance rejection capabilities of the equivalent delay-free loop are preserved. In order to place this contribution in context, some modifications of the SP are revisited and recast under the same structure. The features of the proposed scheme are illustrated through simulations, showing a comparison with respect to the corresponding delay-free loop, which is here considered to be the ideal scenario. In order to emphasize the feasibility of this approach, a successful experimental implementation in a laboratory platform is also reported.
\end{abstract}

Keywords: Smith Predictor, time delay, external disturbances, single-input single-output system

\section{Introduction}

Time delays appear naturally in control applications. They can be either intrinsic to the physical process to be controlled or originated in the implementation of the feedback loop [1]. Furthermore, industrial processes usually operate in a fixed setpoint during long periods of time and thus disturbance rejection is a fundamental issue.

An LTI time-delay SISO process subject to input disturbances can be described by

$$
y(s)=G(s) e^{-h s}[u(s)+w(s)] \triangleq \bar{y}(s) e^{-h s}
$$

where $y \in \mathbb{R}$ is the measurable output, $\bar{y} \in \mathbb{R}$ is the unmeasurable non-delayed output, $u \in \mathbb{R}$ is the control input, $w \in \mathbb{R}$ is an input disturbance, $h \geq 0$ is a constant time delay and $G(s)=C(s I-A)^{-1} B$ is referred to as the delay-free system.

When controlling a time-delay system, an ideal scenario is depicted in Fig. 1. It is "ideal" in the sense that the delay is pushed out of the feedback loop, the non-delayed output $\bar{y}$ is available, and thus the controller $K(s)$ can be simply designed for the rational part of the model, $G(s)$, using conventional techniques. Since $\bar{y}$ is not accessible, a reasonable approach consists of constructing an output prediction $\hat{\bar{y}}$, so that it can be used to control the system as in the ideal scenario. The prediction should be based on the available input/output information, having the following structure:

$$
\hat{\bar{y}}(s) \triangleq F_{1}(s) u(s)+F_{2}(s) y(s)
$$

where the filters $F_{1}(s)$ and $F_{2}(s)$ must be stable and derived from the plant model.

\footnotetext{
${ }^{*}$ Corresponding author

Email addresses: risanzdi@gmail .com (R. Sanz), pggil@isa.upv.es (P. García), pedro@aii.upv.es (P. Albertos)
}

In the seminal work [2], the Smith Predictor (SP) makes use of the filter $F_{1}^{\mathrm{SP}}(s) \triangleq G(s)-G(s) e^{-s h}$, sometimes referred to as the SP block, whereas $F_{2}^{\mathrm{SP}}(s) \triangleq 1$. It is easy to verify that the prediction $\hat{\bar{y}}_{\mathrm{SP}}(s) \triangleq F_{1}^{\mathrm{SP}}(s) u(s)+F_{2}^{\mathrm{SP}}(s) y(s)$ satisfies $\hat{\bar{y}}_{\mathrm{SP}}(s)=G(s) u(s)=\bar{y}(s)$, if there are no disturbances. Indeed, the SP removes the delay element from the denominators of all the closed-loop sensitivity functions, reducing the control problem to that of a delay-free system. The methodology described above has been referred to as the "Smith's Principle" in the literature. However, the SP cannot be applied to open-loop unstable plants and regardless of the main controller, only constant disturbances can be rejected [3].

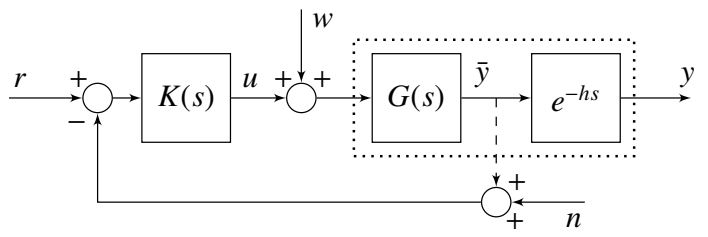

Figure 1: An ideal control loop (unfeasible)

Many structures, commonly referred to as dead-time compensators (DTCs), have been developed to mitigate these issues $[4,5]$, either to achieve load disturbance rejection for pure integrating processes with long dead-time $[6,7,8,9,10,11,12]$, or to control unstable time-delay systems $[13,14,15,16,17,18$, $19,20,21]$. Some works have been also focused on counteracting periodic disturbances [22, 23, 24]. These schemes commonly have an inner stabilizing loop and employ more controllers. Furthermore, most solutions are highly specific on the control goals and/or the plant structure, and they fail in completely removing the delay element from the feedback loop, making the design process more complicated. To the best of 
the author's knowledge, for integrating and unstable systems, none of the aforementioned works except those proposed in $[6,18,19]$, fulfill the Smith's Principle. Next, these schemes are reviewed and recast under the same structure, in order to place the present work in context.

\subsection{The Smith's Principle}

As aforementioned, few schemes have been proposed to generalize the Smith Predictor to unstable systems, avoiding the instability of the predictor block while fulfilling the Smith's Principle. The first attempt in this direction can be found in [6]. In that work, the SP block was modified by choosing $F_{1}^{\mathrm{MSP}}(s) \triangleq G^{\dagger}(s)-G(s) e^{-s h}$ and $F_{2}^{\mathrm{MSP}}(s)=1$, where $G^{\dagger}(s) \triangleq C e^{-A h}(s I-A)^{-1} B$. However, it was later where this approach was generalized and named as the Modified Smith Predictor (MSP) [25]. The key feature of this scheme is that the MSP block can be computed in the time domain as ${ }^{1} \mathcal{L}^{-1}\left\{F_{1}^{\mathrm{MSP}}(s) u(s)\right\}=C e^{-A h} \int_{0}^{h} e^{A \xi} B u(t-\xi) \mathrm{d} \xi$, which is a definite integral and therefore, stable. Regarding disturbance rejection, the MSP alters the low frequency gain of the primary controller because it has non-zero static gain, that is, $F_{1}^{\mathrm{MSP}}(0) \neq 0$. Consequently, constant disturbances cannot be rejected even if the primary controller contains integral action. This drawback was already addressed in [6] by choosing $G^{\dagger}(s)=-C \int_{0}^{h} e^{-A \xi} \mathrm{d} \xi B+C e^{-A h}(s I-A)^{-1} B$, with the inconvenient that $G^{\dagger}(s)$ is no longer strictly-proper and the corresponding controller may be more complicated.

Other proposals were developed inspired on the discrete-time framework. In [19], the SP was complemented with an additional filter, $F_{2}^{\mathrm{FSP}}(s) \triangleq F_{r}(s)$, leading to the Filtered Smith Predictor (FSP). The resulting predictor block was $F_{1}^{\mathrm{FSP}}(s) \triangleq$ $G(s)-G(s) F_{r}(s) e^{-s h}$, where the new filter $F_{r}(s)$ played a key role, being used to avoid the unstable modes in $F_{1}^{\mathrm{FSP}}(s)$. In continuous-time, this pole-zero cancellation cannot be performed by the use of polynomial division because the numerator of $F_{1}^{\mathrm{FSP}}(s)$ is a non-rational expression. However, in the discrete-time framework, this can be done analytically by solving a Diophantine equation. In the same process, the block can be also adjusted to reject any class of disturbances [26, 27].

The Generalized Predictor (GP), originally proposed in [18], was originated from a discrete-time reasoning. However, the formulation next presented is developed in continuoustime for the sake of comparison. Similarly to the MSP, the instability of the GP block was avoided by selecting $F_{1}^{\mathrm{GP}}(s) \triangleq G(s)-G^{*}(s) e^{-s h}$ with $G^{*}(s) \triangleq C e^{A h}(s I-A)^{-1} B$, whereas $F_{2}^{\mathrm{GP}}(s)=G^{*}(s) / G(s)$. As a result, the GP block can be computed as $\mathcal{L}^{-1}\left\{F_{1}^{\mathrm{GP}}(s) u(s)\right\}=C \int_{0}^{h} e^{A \xi} B u(t-\xi) \mathrm{d} \xi$, which is a stable block. In order to cancel the effect of constant disturbances, the GP made use of an extra loop, making the analysis more complicated [28].

The schemes previously reviewed lead to a control structure as depicted in Fig. 2, with filters given in Table 1.

\footnotetext{
${ }^{1}$ Here $\mathcal{L}^{-1}\{\cdot\}$ denotes the inverse Laplace transform operator.
}

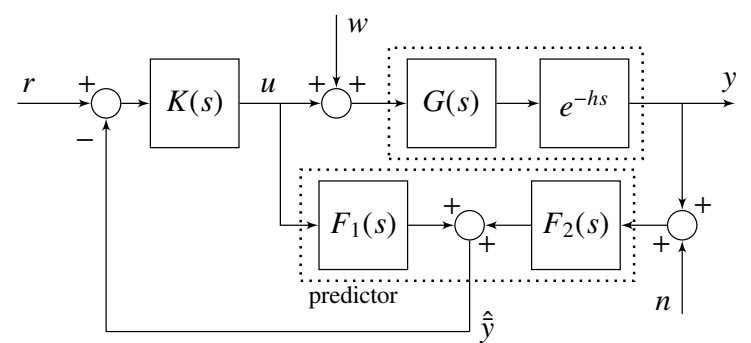

Figure 2: A general structure for predictor-based control schemes

Table 1: Filters in Fig. 2 for each scheme reviewed in Section 1.1

\begin{tabular}{cccc}
\hline Scheme & $F_{1}(s)$ & $F_{2}(s)$ & Proposed in \\
\hline SP & $G(s)-G(s) e^{-s h}$ & 1 & {$[2]$} \\
MSP & $G^{\dagger}(s)-G(s) e^{-s h}$ & 1 & {$[6]$} \\
FSP & $G(s)-G(s) F_{r}(s) e^{-s h}$ & $F_{r}(s)$ & {$[19]$} \\
GP & $G(s)-G^{*}(s) e^{-s h}$ & $G^{*}(s) / G(s)$ & {$[18]$} \\
\hline
\end{tabular}

\subsection{Contribution}

In this paper, with special emphasis on transparency and design simplicity of the resulting control strategy, a generalization of the SP is proposed to solve the following problem:

Problem 1. Consider a controller $K$ designed to meet some requirements based on the delay-free loop depicted in Fig. 1. Then, find a predictor, that is, design filters $F_{1}$ and $F_{2}$, such that the same controller $K$ in Fig. 2:

A) guarantees internal stability

$B$ ) achieves the same nominal tracking performance

$C$ ) achieves rejection of the same type of disturbances

\section{Problem reformulation}

As already mentioned, a celebrated feature of the SP is that it exactly reduces the control problem to its delay-free counterpart, by constructing an "exact" prediction. In what follows, a prediction $\hat{\bar{y}}$ for the system (1) is said to be exact if $\hat{\bar{y}}(s)=\bar{y}(s)$ hold in the nominal case. It is easy to show that a prediction computed by (2) is exact if and only if

$$
F_{1}(s)=\left(1-F_{2}(s) e^{-h s}\right) G(s)
$$

The main advantage of obtaining an exact prediction is that the design and analysis of the resulting control-loop are drastically simplified, which is a highly appreciated feature of the original SP. This is formally stated by the following proposition:

Proposition 1. If the output prediction computed by (2) is exact, then the input-output transfer functions of the predictorbased control loop depicted in Fig. 2 satisfy:

$$
\begin{aligned}
G_{r, y}(s) & =\bar{G}_{r, y}(s) \\
G_{w, y}(s) & =\bar{G}_{w, y}(s)+\bar{G}_{r, y}(s) F_{1}(s) \\
G_{n, y}(s) & =\bar{G}_{n, y}(s) F_{2}(s) \\
G_{n, u}(s) & =\bar{G}_{n, u}(s) F_{2}(s)
\end{aligned}
$$

where $\bar{G}_{r, y}, \bar{G}_{r, w}, \bar{G}_{r, n}, \bar{G}_{n, u}$ are the input-output transfer functions of the ideal loop in Fig. 1. 
Proof. The proposition follows simply by solving the block diagrams in Figs. 1-2 and using (3).

Remark 1. Inspecting Table 1, the condition (3) holds for all the schemes reviewed in Section 1.1 but the MSP, in which the controller has to be designed for the modified plant $G^{\dagger}(s)$. This can be an inconvenient in some cases, as already mentioned.

Now, Problem 1 is translated into finding a predictor with some constraints. To that purpose, let us introduce the following assumption:

Assumption 1. The external disturbance can be expressed as $w(s)=\bar{w} w_{0}(s)$, with unknown amplitude $\bar{w} \in \mathbb{R}$ and known dynamics $w_{0}(s)$

Assumption 1 implies that the type of disturbance to be rejected should be known, e.g., step, ramp, or sine wave with a given frequency. Although this may seem restrictive, some attenuation of disturbances not described by $w_{0}(s)$ is also expected. This can be analyzed in detail by looking at the bode plot of (5).

Lemma 1. Let us consider the control loop in Fig. 2 with a predictor such that:

i.) the prediction $\hat{\bar{y}}$ is exact

ii.) the filters $F_{1}(s), F_{2}(s)$ are stable

iii.) the following equivalent conditions hold

$$
\lim _{t \rightarrow \infty}(\bar{y}(t)-\hat{\bar{y}}(t))=0 \Longleftrightarrow \lim _{s \rightarrow 0} s F_{1}(s) w_{0}(s)=0
$$

Then, that predictor solves Problem 1.

Proof. If i.) holds then Proposition 1 is valid and the control loop in Fig. 2 is internally stable iff (4)-(7), the so-called "gang of four", are stable. Recall that $K(s)$ is designed such that all transfer functions of the ideal loop, denoted with an upper bar are stable, whereas the filters are stable by ii.). Then A) in Problem 1 is fulfilled. Also, the set-point responses in (4) for both loops are the same and thus B) is fulfilled. Finally, using (1)-(3), the output prediction error due to the input disturbance satisfies

$$
e(s) \triangleq \bar{y}(s)-\hat{\bar{y}}(s)=F_{1}(s) w(s)
$$

The equivalence in iii.) follows from (8) and Assumption 1. Furthermore, notice that if iii.) holds, from (5), the load disturbance response of the ideal loop is recovered in steady-state, and then, C) is fulfilled. This completes the proof.

\section{Proposed generalized SP}

The main contribution of this paper, a generalized SP, is presented next.

Lemma 2 (GSP). Let us consider an arbitrary decomposition of the delay-free plant such that

$$
G(s)=\Gamma(s) \tilde{G}(s)
$$

where $\Gamma(s)$ is proper, stable and may have non-minimum phase zeros; and $\tilde{G}(s)=\tilde{C}(s I-\tilde{A})^{-1} \tilde{B}=\tilde{N}(s) / \tilde{D}(s)$ is strictly proper, minimum phase and may have unstable poles. Then, the computation of (2) with the stable filters

$$
F_{1}(s)=\Phi_{\tilde{G}}(s) \Gamma(s) \quad F_{2}(s)=\frac{\tilde{N}^{*}(s)}{\tilde{N}(s)}
$$

where $\tilde{G}^{*}(s)=\tilde{C} e^{\tilde{A} h}(s I-\tilde{A})^{-1} \tilde{B}=\tilde{N}^{*}(s) / \tilde{D}(s)$ and $\Phi_{\tilde{G}}(s)=$ $\tilde{C}\left(I-e^{-(s I-\tilde{A}) h}\right)(s I-\tilde{A})^{-1} \tilde{B}$, provides an exact output prediction.

Proof. See Appendix A.

Intuitively, Lemma 2 implies that, regarding prediction, the plant can be decomposed into: $\tilde{G}(s)$, which is projected $h$ units of time ahead by the operator $\Phi_{\tilde{G}}(s)$; and $\Gamma(s)$, which appears explicitly in the predictor. The usefulness of the GSP introduced above lies on the fact that the prediction is exact no matter what decomposition is chosen. Therefore, $\Gamma(s)$ can be appropriately selected so that $F_{1}(s)$ has some desired properties.

Theorem 1. Let us consider the following decomposition

$$
\Gamma(s)=\frac{N^{+}(s) N_{\Gamma}^{-}(s)}{D_{\Gamma}^{-}(s)} \frac{Q(s)}{w_{0}(s)} \quad \tilde{G}(s)=\frac{N_{\tilde{G}}^{-}(s)}{D^{+}(s) D_{\tilde{G}}^{-}} \frac{w_{0}(s)}{Q(s)}
$$

where: $i$.) the unstable poles and non-minimum phase zeros of $G(s)$ are collected in $D^{+}(s)$ and $N^{+}(s)$, respectively; ii.) its stable poles $D^{-}(s)$, and minimum phase zeros $N^{-}(s)$, are arbitrarily partitioned so that $D_{\Gamma}^{-}(s) D_{\tilde{G}}^{-}(s)=D^{-}(s)$ and $N_{\Gamma}^{-}(s) N_{\tilde{G}}^{-}(s)=N^{-}(s)$; and iii.) $Q(s)$ is a strictly-proper filter such that $\tilde{G}(s)$ is strictly-proper and $\Gamma(s)$ is at least proper.

Then, the GSP introduced in Lemma 2 with $\Gamma(s), \tilde{G}(s)$ given above, solves Problem 1.

Proof. Recall that Problem 1 is solved if the conditions in Lemma 1 are fulfilled. By Lemma 2, the items i.) and ii.) hold. Using (8), the limit of the output prediction error (assuming it exists) can be computed as $\lim _{t \rightarrow \infty} e(t)=\lim _{s \rightarrow 0} s F_{1}(s) w(s)$. Using (10), (11) and Assumption 1, it follows that

$$
\begin{aligned}
\lim _{t \rightarrow \infty} e(t) & =\lim _{s \rightarrow 0} s \Phi_{\tilde{G}}(s) \frac{N^{+}(s) N_{\Gamma}^{-}(s)}{D_{\Gamma}^{-}(s)} \frac{Q(s)}{w_{0}(s)} \bar{w} w_{0}(s) \\
& =\lim _{s \rightarrow 0} s \Phi_{\tilde{G}}(s) \frac{N^{+}(s) N_{\Gamma}^{-}(s)}{D_{\Gamma}^{-}(s)} Q(s) \bar{w}=0
\end{aligned}
$$

Notice that the limit always exists because $\Phi_{\tilde{G}}(0)$ is well defined and the other transfer functions in (12) have stable poles. This completes the proof.

Remark 2. It should be remarked that although there are infinite choices for $Q(s)$, its relative degree, denoted by $r_{q}$, is constrained. In order to fulfill the third condition in Theorem 1, it can be shown that $r_{q} \in\left[\underline{r}_{q}, \bar{r}_{q}\right] \triangleq\left[z^{+}+r_{w_{0}}-p^{-}, p+r_{w_{0}}-1\right]$, where $r_{w_{0}}$ is the relative degree of $w_{0}(s), z^{+}$is the number of non-minimum phase zeros, $p$ is the number of total poles and $p^{-}$is the number of stable poles. A simple choice is $Q(s)=(s+\omega)^{-r_{q}}, r_{q} \in\left[\underline{r}_{q}, \bar{r}_{q}\right]$, which leaves only two parameters to be adjusted. 


\section{Predictor-based control}

The main features of the control-loop resulting from applying the proposed predictor are discussed in this section. First, performance and robustness indices are derived in comparison to those of the ideal delay-free loop. Later, the stability of the digital implementation is thoroughly analyzed as it is a critical issue, specially for unstable plants.

\subsection{Performance and robustness}

From (4) in Proposition 1, the proposed strategy recovers the tracking performance of the ideal loop. Also, it can be verified from (5) that

$$
S(s)=\bar{S}(s)\left(1+K(s) F_{1}(s)\right)
$$

where $S(s)$ and $\bar{S}(s)$ are the sensitivity functions of the control loops in Fig. 1 and Fig. 2, respectively. On the other hand, let us consider a multiplicative uncertainty such that $G(s)=G_{0}(s)\left(1+\Delta(s) W_{\Delta}(s)\right)$ with $\|\Delta\|_{\infty} \leq 1$, and thus the robust stability condition for Fig. 1 is $\|\bar{M}(s)\|_{\infty} \leq 1$ where $\bar{M}=\bar{L}(s) W_{\Delta}(s) /(1+\bar{L}(s))$ and $\bar{L}(s)=G_{0}(s) K(s)$. Then, denoting by $\|M(s)\|_{\infty} \leq 1$ the new robust stability condition for the loop Fig. 2, it can be show that

$$
M(s)=\bar{M}(s) F_{2}(s)
$$

The robustness with respect to a delay mismatch is also a matter of concern [29]. Considering $h=h_{0}+\delta h$, the robust stability can be checked by modeling it as multiplicative uncertainty (very conservative), or the analytic bounds on $\delta h$ can be computed by

$$
\max \left\{-h_{0}, \frac{\phi_{i}^{-}}{\omega_{i}}\right\}<\delta h<\min \frac{\phi_{i}^{+}}{\omega_{i}},
$$

where $\omega_{i}$ are crossover frequencies at which $L\left(j \omega_{i}\right)=1$, $\phi_{i}^{+}>0, \phi_{i}^{-}<0$ are the corresponding signed phase margins, and $L(s)$ is the loop transfer function of Fig. 2, given by

$$
L(s)=\frac{G(s) K(s) F_{2}(s) e^{-h s}}{1+K(s) F_{1}(s)} .
$$

Since $K(s)$ should be designed for the delay-free system, one can use (13)-(14) along with Theorem 1 to design a filter $Q(s)$ so that the desired disturbance rejection performance and robustness are achieved, if possible. However, it is worth mentioning that there are fundamental limitations [30]. For example, it is not possible to achieve $\bar{S}(s)=S(s)$ at all frequencies because $F_{1}(s)=0$ implies $F_{2}(s)=e^{s h}$, which is not realizable. Also, $\bar{M}(s)=M(s)$ can only be achieved for stable systems, because it implies that $F_{2}(s)=1$ and thus $F_{1}(s)=G(s)\left(1-e^{-h s}\right)$, which is the conventional Smith Predictor. It should be also remarked that, although predictors achieve nominal stability for any $h>0$, there is a limitation on the achievable delay margin for unstable systems using LTI controllers [31]. Therefore, above that value, an infinitesimal delay would lead to instability, making the controller unfeasible in practice.

\subsection{Discrete-time implementation}

Some details regarding the digital implementation will be discussed next. Since predictive schemes are sensitive to nonminimum phase zeros and unstable poles, it is important to consider a discretized plant from the beginning. Let us define the sampling period $T_{s}>0$, and the discretized process model $G(z)=\mathcal{Z}\{G(s)\}$, where $\mathcal{Z}\{\cdot\}$ is the $Z$-transform operator. The delay is assumed to be a multiple of the sampling period, i.e., $h=T_{s} d$ for some $d \in \mathbb{N}$. Then, the discrete-time counterpart of (1) is given by

$$
y(z)=G(z) z^{-d}[u(z)+w(z)]
$$

where $G(z)=C_{z}\left(z I-A_{z}\right)^{-1} B_{z}$. The discrete form of the proposed predictor for the system (16) is introduced in the following lemma:

Lemma 3 (Discrete-time GSP). Let us consider an arbitrary decomposition of the delay-free plant such that

$$
G(z)=\Gamma(z) \tilde{G}(z)
$$

where $\Gamma(z)$ is proper, stable and may have non-minimum phase zeros; and $\tilde{G}(z)=\tilde{C}_{z}\left(z I-\tilde{A}_{z}\right)^{-1} \tilde{B}_{z}=\tilde{N}(z) / \tilde{D}(z)$ is strictly proper, minimum phase and may have unstable poles. Then, the computation of

$$
\hat{\bar{y}}(z)=F_{1}(z) u(z)+F_{2}(z) y(z)
$$

with the stable filters

$$
F_{1}(z)=\Phi_{\tilde{G}}(z) \Gamma(z) \quad F_{2}(z)=\frac{\tilde{N}^{*}(z)}{\tilde{N}(z)}
$$

where $\tilde{G}^{*}(z)=\tilde{C}_{z} \tilde{A}_{z}^{d}\left(z I-\tilde{A}_{z}\right)^{-1} \tilde{B}_{z}=\tilde{N}^{*}(z) / \tilde{D}(z)$ and $\Phi_{\tilde{G}}(z)=$ $\tilde{C}_{z} \sum_{j=1}^{d} \tilde{A}_{z}^{j-1} z^{-j} \tilde{B}_{z}$, provides an exact output prediction.

Proof. It can be readily seen that the following identity holds $\sum_{j=1}^{d} \tilde{A}_{z}^{j-1} z^{-j}=\left(I-\tilde{A}_{z}^{d} z^{-d}\right)\left(z I-\tilde{A}_{z}\right)^{-1}$. Pre-multiplying by $\tilde{C}_{z}$ and post-multiplying by $\tilde{B}_{z}$ in the previous identity yields $\Phi_{\tilde{G}}(z)=\tilde{G}(z)-\tilde{G}^{*}(z) z^{-d}$. Therefore, from (19), if follows that $F_{1}(z)=\left(\tilde{G}(z)-\tilde{G}^{*}(z) z^{-d}\right) \Gamma(z)$. Also, since $\tilde{G}(z)$ and $\tilde{G}^{*}(z)$ have the same poles, then $F_{2}(z)=\tilde{N}^{*}(z) / \tilde{N}(z)=\tilde{G}^{*}(z) / \tilde{G}(z)$. Plugging these expressions into (18) and after some manipulations, it follows that $\hat{\bar{y}}(z)=G(z) u(z)$ for the nominal case, i.e., $w=0$. This completes the proof.

Remark 3. Notice that the decomposition introduced in Theorem 1 applies to the discrete-time case, simply replacing the argument $s$ of the transfer functions by $z$. It is important to perform the decomposition of the plant using the discretized model, because additional zeros may be introduced during the discretization process.

Remark 4. The digital implementation of the distributed terms arisen in $F_{1}(s)$ has been a major concern for many years [32]. The implementation structure given in Lemma 3 mitigates this problem. Another implementation issue was devised in [33] for the case of systems with fast stable modes, where the Unified Smith Predictor (USP) was proposed. This problem can be avoided in the proposed scheme by placing the fast stable modes in $\Gamma(s)$ and selecting $Q(s)$ accordingly. 
Table 2: Absolute performance and robustness for different values of $\tau_{q}$

\begin{tabular}{rccccc} 
& $G M$ & $P M$ & $I A E / I A E^{*}$ & $\omega_{c}$ & $\delta h$ \\
\hline \hline delay-free & {$[0.79$, inf $]$} & $35.9^{\circ}$ & 1 & $1.3 \mathrm{rad} / \mathrm{s}$ & - \\
\hline$\tau_{q}=0.1 \mathrm{~s}$ & {$[0.83,1.20]$} & $11.5^{\circ}$ & 1.04 & $51.3 \mathrm{rad} / \mathrm{s}$ & $\pm 0.02 \mathrm{~s}$ \\
$\tau_{q}=0.75 \mathrm{~s}$ & {$[0.87,1.18]$} & $7.71^{\circ}$ & 1.07 & $11.4 \mathrm{rad} / \mathrm{s}$ & $\pm 0.09 \mathrm{~s}$ \\
$\tau_{q}=1.5 \mathrm{~s}$ & {$[0.89,1.18]$} & $6.4^{\circ}$ & 1.12 & $8.5 \mathrm{rad} / \mathrm{s}$ & $\pm 0.12 \mathrm{~s}$ \\
$\tau_{q}=2 \mathrm{~s}$ & {$[0.91,1.18]$} & $5.9^{\circ}$ & 1.20 & $7.8 \mathrm{rad} / \mathrm{s}$ & $\pm 0.13 \mathrm{~s}$ \\
$\tau_{q}=5 \mathrm{~s}$ & {$[0.94,1.18]$} & $5.0^{\circ}$ & 2.10 & $6.5 \mathrm{rad} / \mathrm{s}$ & $\pm 0.15 \mathrm{~s}$ \\
\hline \hline
\end{tabular}

For the convenience of potential users, the set-up of the proposed strategy can be summarized as follows: 1.) Obtain a discrete-time model of the plant as shown in (16); 2.) Design a primary controller $K(z)$, for the delay-free system (using conventional design techniques); 3.) Find a suitable decomposition $G(z)=\Gamma(z) \tilde{G}(z)$ satisfying the conditions in Theorem 1 (choosing $Q(z)$ as simple as possible, e.g., a low-pass filter, for design simplicity); 4.) Construct the filters $F_{1}(z), F_{2}(z)$ and implement the output predictor $\hat{y}(z)$ as described in Lemma $3 ;$ 5.) Use $\hat{y}(z)$ as the input to the controller; and 6.) Adjust the parameters in $Q(z)$ to reach a trade-off between performance and robustness.

\section{Simulations}

In this section, simulations are carried out to validate the proposed strategy. Let us consider (1), being

$$
G(s)=\frac{1}{s-1} \quad \text { and } \quad h=1.5 \mathrm{~s} .
$$

Remark 5. It should be remarked that this is a rather challenging example. To the best of the authors' knowledge, this system has not been robustly controlled with such a large delay [4]. Furthermore, it is pointed out in [31] that no LTI controller can stabilize this system for delays $h>2 s$.

The equivalent $\mathrm{ZOH}$ sampled system (16), with a sampling period $T_{s}=0.01 \mathrm{~s}$ is obtained as $G(z)=b_{z} /\left(z-a_{z}\right)$ with $a_{z}=e^{T_{s}}, b_{z}=\int_{0}^{T_{s}} e^{\theta} \mathrm{d} \theta=1-e^{T_{s}}$ and a discrete delay $d=150$. The controllers below are designed taking into account only the delay-free system, using conventional procedures. The predictor is adjusted to yield zero steady-state prediction error for some type of disturbances. Then it is showed how the straightforward combination of the predictor with the main controller stabilizes the delayed system, keeping the same tracking performance while maintaining the disturbance rejection capabilities.

\subsection{Constant disturbance rejection}

Rejection of constant disturbances is a typical requirement in practice, and it is here chosen to illustrate the main features of the proposed strategy. Primary controller design: A simple 2-DoF PI-controller $K(s)$ with a set-point filter $F_{r}(s)$ is designed, in the Laplace domain for convenience, for the equivalent delay-free system as follows

$$
K(s)=k \frac{t_{i} s+1}{t_{i} s} \quad F_{r}(s)=G_{r}(s) / T(s)
$$

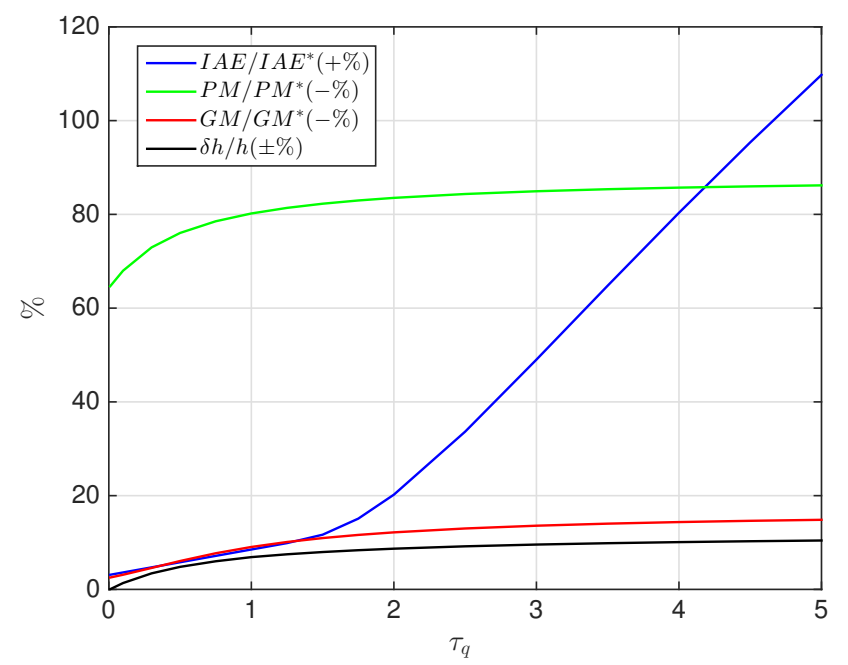

Figure 3: Relative robustness and performance indices as a function of $\tau_{q}$

with $k=2 \beta+1$ and $t_{i}=k / \beta^{2}$, which yields a closed-loop characteristic polynomial $(s+\beta)^{2}$. For the prefilter,

$$
T(s)=\frac{G(s) K(s)}{1+G(s) K(s)}, \quad G_{r}(s)=\frac{1}{\tau_{r} s+1},
$$

leading to a characteristic response time for the set-point tracking, $\tau_{r}$. For the simulations below, the parameters $\beta=1 / 8$ and $\tau_{r}=2 \mathrm{~s}$ are arbitrary selected. Notice that this controller is able to reject constant disturbances in the delay-free case and it has been designed without considering the time delay. Predictor design: The predictive scheme is implemented according to Lemma 3, with $N_{\Gamma}^{-}(z)=b_{z}, N_{\tilde{G}}^{-}(z)=1, N^{+}(z)=1$, $D^{+}(z)=\left(z+a_{z}\right), D_{\Gamma}^{-}(z)=1, D_{\tilde{G}}^{-}(z)=1$ and $w_{0}(s)=1 / s$, to reject constant disturbances. Notice that according to Remark 1 , in this case $r_{q} \in[1,1]$ and thus the simplest possible choice for the filter is taken, $Q(s)=1 /\left(\tau_{q} s+1\right)$, being $\tau_{q}$ an adjustable parameter. The following discretization can be obtained

$$
\mathcal{Z}\left\{\frac{Q(s)}{w_{0}(s)}\right\}=\mathcal{Z}\left\{\frac{s}{\tau_{q} s+1}\right\}=\frac{z-1}{\tau_{q} z+\left(T_{s}-\tau_{q}\right)}
$$

and thus, applying the decomposition in Theorem 1 yields

$$
\Gamma(z)=\frac{b_{z}(z-1)}{\tau_{q} z+\left(T_{s}-\tau_{q}\right)} \quad \text { and } \quad \tilde{G}(z)=\frac{\tau_{q} z+\left(T_{s}-\tau_{q}\right)}{(z-1)\left(z-a_{z}\right)} .
$$

A state-space realization of $\tilde{G}(z)=\tilde{C}_{z}\left(z I-\tilde{A}_{z}\right)^{-1} \tilde{B}_{z}$ is given by

$$
\tilde{C}_{z}=\left[T_{s}-\tau_{q}, \tau_{q}\right], \quad \tilde{A}_{z}=\left[\begin{array}{cc}
0 & 1 \\
1+a_{z} & -a_{z}
\end{array}\right] \quad \text { and } \quad \tilde{B}_{z}=\left[\begin{array}{l}
0 \\
1
\end{array}\right] \text {. }
$$

According to Lemma 3, the filter $F_{1}(z)$ can be computed by

$$
F_{1}(z)=\Phi_{\tilde{G}}(z) \frac{b_{z}(z-1)}{\tau_{q} z+\left(T_{s}-\tau_{q}\right)}
$$

with $\Phi_{\tilde{G}}(z)=\tilde{C}_{z} \sum_{j=1}^{d} \tilde{A}_{z}^{j-1} z^{-j} \tilde{B}_{z}$ and $\tilde{A}_{z}, \tilde{B}_{z}, \tilde{C}_{z}$ given above. In order to compute $F_{2}(z)$, notice that, because of the canonical form of the state-space representation of $\tilde{G}(z)=\tilde{N}(z) / \tilde{D}(z)$, it 

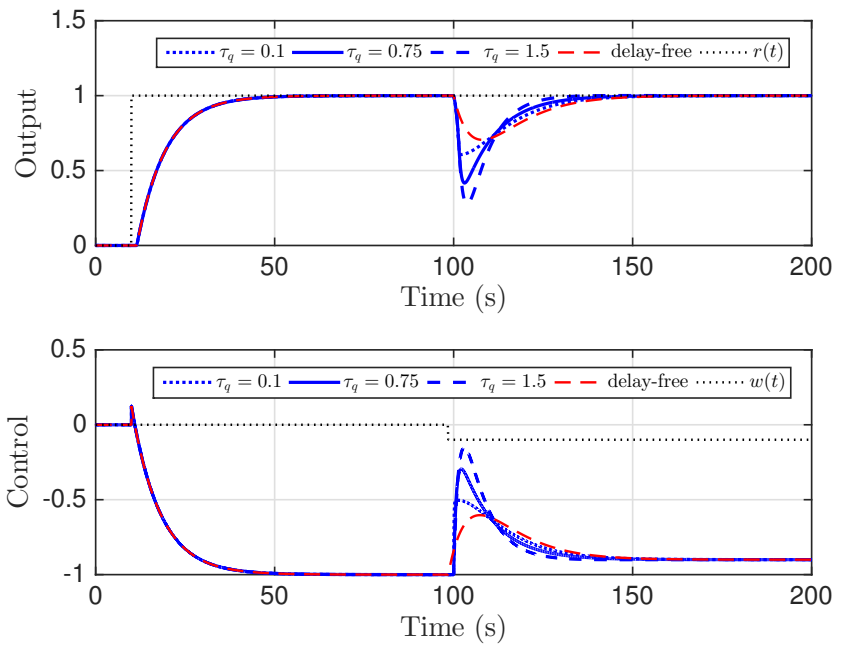

Figure 4: Nominal performance for different values of $\tau_{q}$

follows that $\tilde{N}(z)=\tau_{q} z+\left(T_{s}-\tau_{q}\right)=\left\langle\tilde{C}_{z},[1, z]\right\rangle$, where $\langle\cdot, \cdot\rangle$ denotes the dot product. In other words, the coefficients of the numerator of the transfer function are simply given by $\tilde{C}_{z}$. Then, according to Lemma 3 , the numerator of $\tilde{G}^{*}(z)$ is obtained as $\tilde{N}^{*}(z)=\left\langle\tilde{C}_{z} \tilde{A}_{z}^{d},[1, z]\right\rangle$ and thus the filter $F_{2}(z)$ can be computed by

$$
F_{2}(z)=\frac{\left\langle\tilde{C}_{z},[1, z]\right\rangle}{\left\langle\tilde{C}_{z} \tilde{A}_{z}^{d},[1, z]\right\rangle}
$$

In the current configuration, there is only one parameter left to be tuned, namely, $\tau_{q}$. Its influence can be illustrated through a plot like the one depicted in Fig. 3. In this representation, one can see the phase margin reduction (green), the gain margin reduction (red) and the integral absolute error (IAE) increment for a load step disturbance (blue), all of them expressed as relative values over the corresponding delay-free loop characteristics. According to the previous indicators, lower values of $\tau_{q}$ increase both robustness and performance. However, the allowable delay uncertainty (black) approaches to zero as $\tau_{q} \rightarrow 0$. The data shown in Table 2 (absolute GM, absolute PM, relative IAE and absolute delay mismatch, $\delta h$ ) illustrates with more details the same behavior depicted in Fig. 3. Furthermore, an additional measure is added, namely, the crossover frequency of the transfer function (6), denoted by $\omega_{c}$. It can be concluded that the improvement in GM, PM and IAE comes at the expense of more noise and less tolerance to delay mismatch.

Therefore, a trade-off must be reached, which is mainly constrained by how accurate the delay is known. From Fig. 3, for this particular example, values $\tau_{q}>2$ should be discarded, as they reduce performance substantially (fast grow of relative IAE) whereas the other indices are barely improved. On the other hand, values in the range $\tau_{q}<0.5$ offer a tolerance of less than $5 \%$ to a delay mismatch, and thus they should also be ruled out. An interval of interest in practice for this example is hence given by $0.5<\tau_{q}<2$.

The discussion above is illustrated next through some simulations. The system is driven by a step reference $r(t)=1, \forall t \geq 10 \mathrm{~s} ;$ and an input disturbance
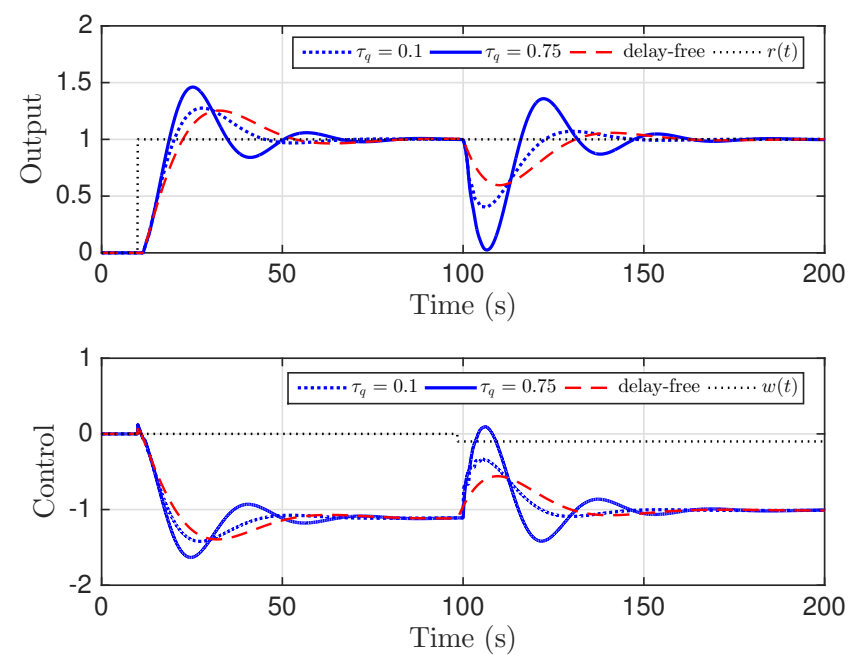

Figure 5: Robustness to $-10 \%$ gain variation for different values of $\tau_{q}$
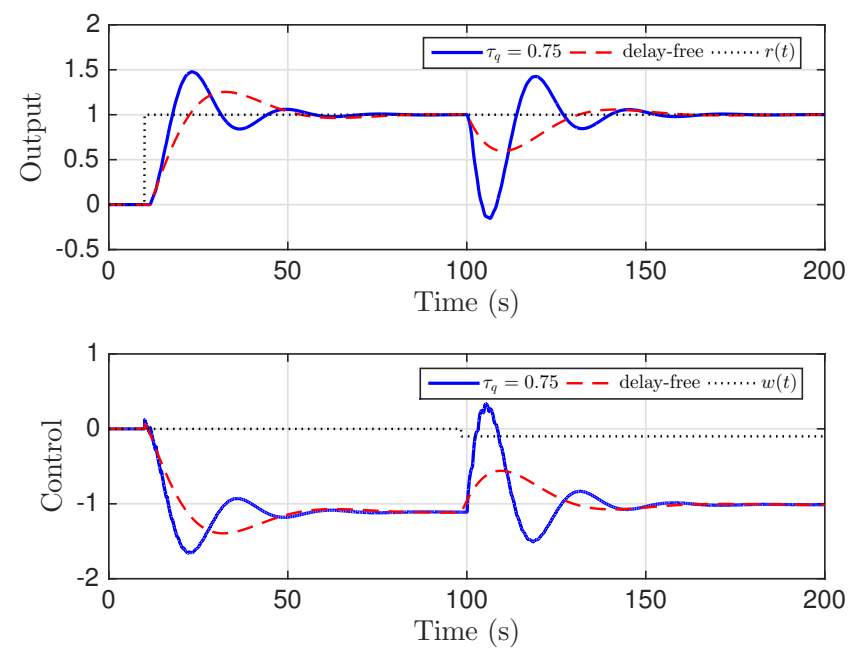

Figure 6: Robustness to $-10 \%$ gain and $+5 \%$ delay variations

$w(t)=-0.1, \forall t \geq 100 \mathrm{~s}$ is applied. The first scenario is shown in Fig. 4, where the different values of $\tau_{q}$ are simulated in nominal conditions. One can clearly see how the disturbance rejection performance improves as $\tau_{q}$ is reduced. A second simulation shows the effect of $\tau_{q}$ in the robust performance. The gain of the actual plant is decreased by $10 \%$ while the delay is kept with its nominal value. The results are shown in Fig. 5. It is verified that, as discussed before, if the delay is accurately known, lower values of $\tau_{q}$ provide better robustness. The output corresponding to $\tau_{q}=1.5 \mathrm{~s}$ is not included here because it is almost unstable. The third simulation considers the same scenario as before but the actual delay is increased by $5 \%$. In this case, the value $\tau_{q}=0.1 \mathrm{~s}$ leads to instability, as it has a very small tolerance to a delay mismatch (see Table 2). However, the performance for $\tau_{q}=0.75 \mathrm{~s}$, shown in Fig. 6, is satisfactory.

\subsection{Sinusoidal disturbance rejection}

The design process to reject a sinusoidal input disturbance is illustrated next. A resonant controller $K(s)$ with a set-point 

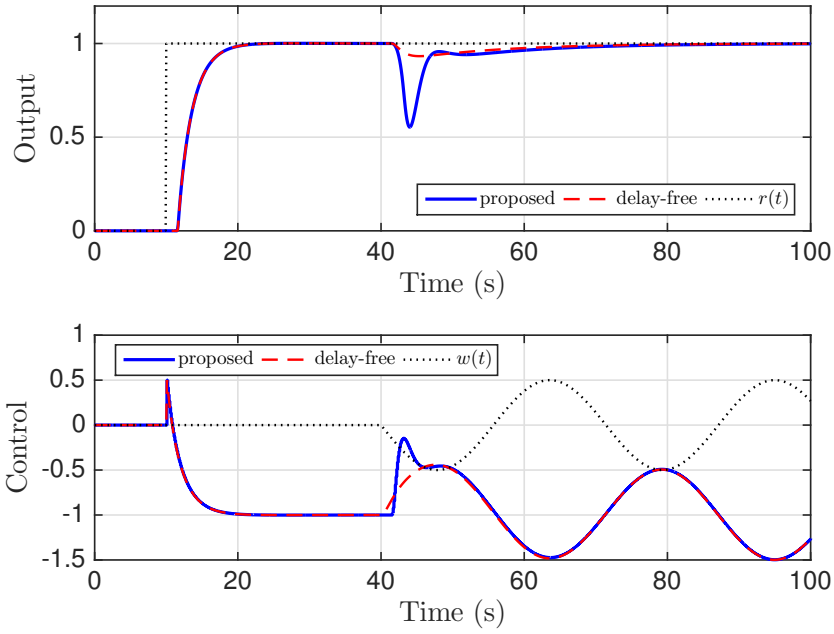

Figure 7: Rejection of a sinusoidal input disturbance

filter $F_{r}(s)$ is designed for the equivalent delay-free system as follows

$$
K(s)=k \frac{(s+\omega)^{2}}{s^{2}+\omega^{2}} \quad F_{r}(s)=G_{r}(s) / T(s)
$$

with $k=5, \omega=0.2 \mathrm{rad} / \mathrm{s}$ which yields a stable closed-loop with phase margin $60^{\circ}$. The prefilter is the same as in the previous example, with $\tau_{r}=2 \mathrm{~s}$. Notice that this controller is able to reject sinusoidal disturbances of frequency $\omega=0.5 \mathrm{rad} / \mathrm{s}$ in the delay-free case. The predictive scheme is implemented according to Lemma 3, with the same decomposition as in the previous example but choosing $w_{0}(s)=\omega /\left(s^{2}+\omega^{2}\right)$. Again, in this case $r_{q} \in[2,2]$ according to Remark 1 , and thus the simple choice $Q(s)=1 /\left(\tau_{q} s+1\right)^{2}$ is made, being $\tau_{q}$ an adjustable parameter.

The simulation is shown in Fig. 7, where the adjustable parameter is selected as $\tau_{q}=1 \mathrm{~s}$; the system is driven by a step reference $r(t)=1, \forall t \geq 10 \mathrm{~s}$; and an input disturbance $w(t)=-0.2 \sin 0.2(t-40), \forall t \geq 40 \mathrm{~s}$ is applied. It can be seen how the disturbance is finally rejected in spite of the large time delay, as it happens in the delay-free case. Regardless of whether the frequency of sinusoidal disturbances is accurately known in practice, the main purpose of the previous simulation is to show that the proposed predictor can be easily adjusted to keep the same steady-state disturbance rejection capabilities of the corresponding delay-free loop.

\section{Experimental results}

An experimental validation is reported using the 3D Hover laboratory platform manufactured by Quanser ${ }^{\odot}$, depicted in Fig. 8. It consists of a quadrotor mounted on a 3-DoF pivot joint so that the body can freely rotate in roll, pitch and yaw. The angular position is the controlled variable, which is measured by a encoder with a resolution of $0.04 \mathrm{deg}$, while the input is the voltage sent to the motors. The experiment is performed in one of the roll/pitch axes, which is modeled as a double integrator $G(s)=0.1 / s^{2}$. The control loop is implemented at $T_{s}^{-1}=100 \mathrm{~Hz}$, where $T_{s}$ is the sampling period,

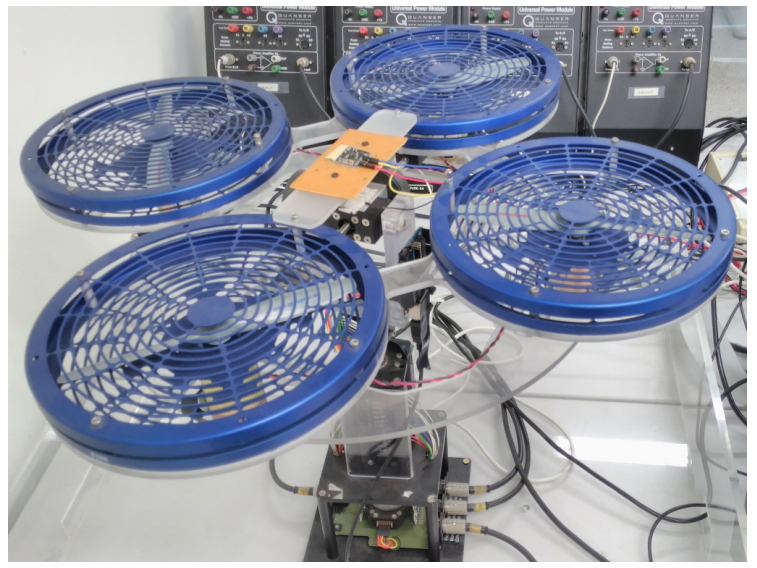

Figure 8: Experimental device

using a POSIX thread in a computer running Linux with a soft real-time patched kernel. An artificial delay of $h=250 \mathrm{~ms}$ (or $d=25$ sampling periods) is introduced by software. The resulting controller is required to reject step disturbances. It should be remarked that the double integrator model is just an approximation of the real plant. In fact, the experimental device has a large uncertainty due to the motor dynamics, which is neglected in the design process. Furthermore, although the number of samples in the artificial delay is known, the actual delay depends on the computational time, which is slightly varying and thus, another source of uncertainty.

Following the Step 1, described at the end of Section 4, a discretization of the plant, $G(z)=0.5 T_{s}^{2}(z+1) /(z-1)^{2}$, is obtained. In the Step 2, a primary PID-controller is designed

$$
K(s)=k_{p}\left(1+\frac{t_{d} s}{\tau_{f} s+1}+\frac{1}{t_{i} s}\right)
$$

with a set-point filter $F_{r}(s)=1 /\left(\tau_{r} s+1\right)$ and $\tau_{r}=2 \mathrm{~s}, k_{p}=$ $50 \mathrm{~V} / \mathrm{rad}, t_{d}=t_{i}=0.5 \mathrm{~s}$, with $\tau_{f}=0.2$, which leads to a large phase margin of $80^{\circ}$ and fast step disturbance rejection for the delay-free system. Following the Steps 3-4, the predictor is implemented according to Lemma 3 , with $N_{\Gamma}^{-}(z)=0.5 T_{s}^{2}$, $N_{\tilde{G}}^{-}(z)=1, N^{+}(z)=z+1, D^{+}(z)=(z-1)^{2}, D_{\Gamma}^{-}(z)=D_{\tilde{G}}^{-}(z)=$ 1. Notice that the zero $(z+1)$ is treated as a non-minimum phase term in order to avoid numerical issues. According to Remark 2, the relative degree of $Q(s)$ needs to be $r_{q}=2$, and thus

$$
\frac{Q(z)}{w_{0}(z)}=\mathcal{Z}\left\{\frac{s}{\left(\tau_{q} s+1\right)^{2}}\right\}=\frac{T(z-1) e^{-T / \tau_{q}}}{\tau_{q}^{2}\left(z-e^{-T / \tau_{q}}\right)^{2}} .
$$

The parameter $\tau_{q}$ is tuned online and finally set to $\tau_{q}=0.25 \mathrm{~s}$. Two experiments with the same pattern are carried out. A step reference of $5 \mathrm{deg}$ is commanded at $t=1 \mathrm{~s}$ and an input disturbance of $-4 \mathrm{~V}$ is applied at $t=20 \mathrm{~s}$. One can see in Fig. 9 that, the designed PID-controller in combination with the proposed predictor stabilizes the system and rejects load disturbances (full blue). An experiment without the predictor is also reported, simply to illustrate that this delay is large enough to be considered, as the designed PID-controller cannot stabilize the system by itself (dashed red). 

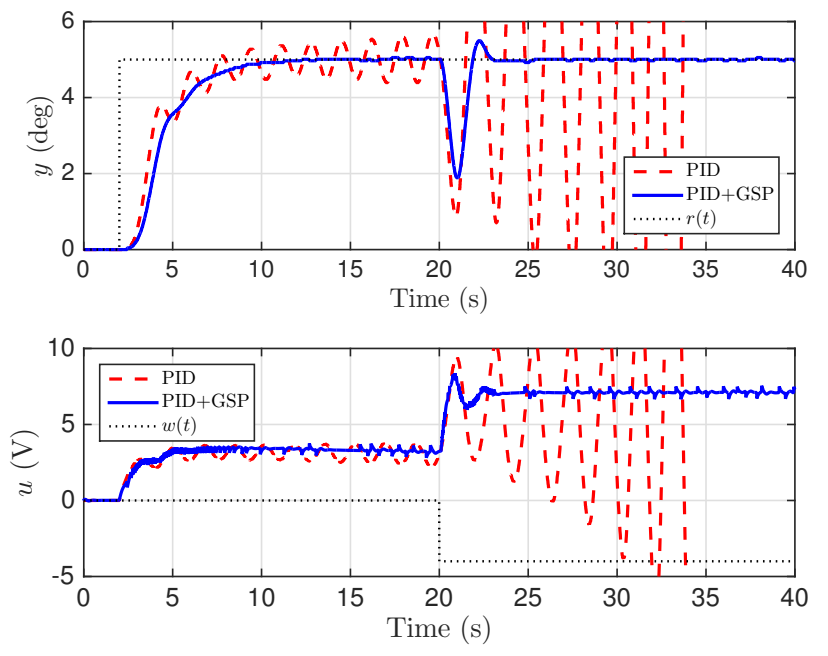

Figure 9: Experimental results of the proposed scheme applied to an unstable plant with a delay of $h=250 \mathrm{~ms}$

\section{Conclusions}

A generalization of the Smith Predictor has been proposed, which retains the main features of the SP but is also applicable to stable/unstable minimum/non-minimum systems. Furthermore, the predictor can be easily adjusted so that the steadystate disturbance rejection capabilities of the primary controller are preserved. The design process is thus drastically simplified. In the simplest configuration, the predictor can be adjusted with just one parameter that can be easily tuned to reach a trade-off between disturbance rejection performance and robustness, while the parameters of the primary controller can be tuned using conventional delay-free techniques. The discretetime implementation, which is usually a handicap, has been also addressed, and its feasibility has been demonstrated with experiments using an unstable laboratory system.

\section{Appendix A. Proof of Lemma 2}

Let us consider the system (1) with $w(t)=0$ and the decomposition (9). The auxiliary variable $v(t)$ is defined such that $v(s)=\Gamma(s) u(s)$, which implies that $y(s)=\tilde{G}(s) e^{-s h} v(s)$. Let us also introduce the following internal representation

$$
\left\{\begin{array}{l}
\dot{x}(t)=\tilde{A} x(t)+\tilde{B} v(t) \\
y(t)=\tilde{C} x(t-h)
\end{array}\right.
$$

so that $\tilde{G}(s)=\tilde{C}(s I-\tilde{A})^{-1} \tilde{B}$. A non-delayed state prediction is given by

$$
\hat{\bar{x}}(t)=e^{\tilde{A} h} x(t-h)+\int_{0}^{h} e^{\tilde{A} \xi} \tilde{B} u(t-\xi) \mathrm{d} \xi
$$

Since the state is not accessible, an output prediction $\hat{\bar{y}}(t)=$ $\tilde{C} \hat{\bar{x}}(t)$, is sought instead. Using (A.1), it follows that

$$
\hat{\bar{y}}(t)=\tilde{C} e^{\tilde{A} h} x(t-h)+\tilde{C} \int_{0}^{h} e^{\tilde{A} \xi} \tilde{B} v(t-\xi) \mathrm{d} \xi
$$

It can be shown that [34]

$$
\tilde{C} \int_{0}^{h} e^{\tilde{A} \xi} \tilde{B} v(t-\xi) \mathrm{d} \xi=\mathcal{L}^{-1}\left\{\Phi_{\tilde{G}}(s) v(s)\right\}
$$

where $\Phi_{\tilde{G}}(s) \triangleq \tilde{C}\left(I-e^{-(s I-\tilde{A}) h}\right)(s I-\tilde{A})^{-1} \tilde{B}$. Also, since $\mathcal{L}\{x(t-h)\}=(s I-\tilde{A})^{-1} \tilde{B} e^{-s h} v(s)$ and $v(s)=\tilde{G}^{-1}(s) e^{s h} y(s)$, one can write

$$
C e^{\tilde{A} h} x(t-h)=\mathcal{L}^{-1}\left\{\frac{\tilde{G}^{*}(s)}{\tilde{G}(s)} y(s)\right\}
$$

where $\tilde{G}^{*}(s)=\tilde{C} e^{\tilde{A} h}(s I-\tilde{A})^{-1} \tilde{B}$. Plugging (A.3)-(A.4) and $v(s)=\Gamma(s) u(s)$ into (A.2) yields

$$
\hat{\bar{y}}(s)=\mathcal{L}\{\hat{\bar{y}}(t)\}=\frac{\tilde{G}^{*}(s)}{\tilde{G}(s)} y(s)+\Phi_{\tilde{G}}(s) \Gamma(s) u(s)
$$

The lemma follows by using the fact that $\tilde{G}^{*}(s) / \tilde{G}(s)=$ $\tilde{N}^{*}(s) / \tilde{N}(s)$ because the transfer functions $\tilde{G}^{*}(s)$ and $\tilde{G}(s)$ have the same denominator.

\section{Acknowledgment}

This work was partially supported by the projects PROMETEOII/2013/004, Conselleria d'Educació, Generalitat Valenciana; TIN2014-56158-C4-4-P-AR, Ministerio de Economía y Competitividad; and the FPI-UPV 2014 PhD Grant, Universitat Politècnica de València, Spain.

\section{References}

[1] R. Sipahi, S.-I. Niculescu, C. T. Abdallah, W. Michiels, K. Gu, Stability and stabilization of systems with time delay, IEEE Control Systems 31 (1) (2011) 38-65.

[2] O. J. M. Smith, Closer control of loops with dead time, Chemical Engineering Progress 53 (1959) 217-219.

[3] J. L. Guzmán, P. Garcia, T. Hägglund, S. Dormido, P. Albertos, M. Berenguel, Interactive tool for analysis of time-delay systems with dead-time compensators, Control Engineering Practice 16 (7) (2008) 824-835.

[4] Q.-G. Wang, H.-Q. Zhou, Y. Zhang, Y. Zhang, A comparative study on control of unstable processes with time delay, in: Control Conference, 2004. 5th Asian, Vol. 3, IEEE, 2004, pp. 1996-2004.

[5] J. E. Normey-Rico, E. F. Camacho, Dead-time compensators: A survey, Control Engineering Practice 16 (4) (2008) 407-428.

[6] K. Watanabe, M. Ito, A process-model control for linear systems with delay, IEEE Transactions on Automatic control 26 (6) (1981) 1261-1269.

[7] K. J. Åström, C. Hang, B. Lim, A new smith predictor for controlling a process with an integrator and long dead time, IEEE Transactions on Automatic Control 39 (1994) 343-345.

[8] M. R. Matausek, A. Micic, On the modified smith predictor for controlling a process with an integrator and long dead-time, IEEE Transactions on Automatic Control 44 (8) (1999) 1603-1606.

[9] J. Normey-Rico, E. Camacho, A unified approach to design dead-time compensators for stable and integrative process with dead-time, IEEE Transactions on Automatic Control 47 (2002) 299-305.

[10] P. García, P. Albertos, A new dead-time compensator to control stable and integrating processes with long dead-time, Automatica 44 (4) (2008) 1062-1071.

[11] S. Uma, M. Chidambaram, A. S. Rao, Set point weighted modified smith predictor with PID filter controllers for non-minimum-phase (nmp) integrating processes, Chemical Engineering Research and Design 88 (5) (2010) 592-601. 
[12] S. Chakraborty, S. Ghosh, A. K. Naskar, All-PD control of pure integrating plus time-delay processes with gain and phase-margin specifications, ISA Transactions 68 (2017) 203-211.

[13] S. Majhi, D. Atherton, Obtaining controller parameters for a new smith predictor using autotuning, Automatica 36 (2000) 1651-1658.

[14] W. Tan, H. J. Marquez, T. Chen, IMC design for unstable processes with time delays, Journal of Process Control 13 (3) (2003) 203-213.

[15] C. Hang, Q.-G. Wang, X.-P. Yang, A modified smith predictor for a process with an integrator and long dead time, Industrial \& Engineering Chemistry Research 42 (3) (2003) 484-489.

[16] T. Liu, W. Zhang, D. Gu, Analytical design of two-degree-of-freedom control scheme for open-loop unstable processes with delay, Journal of Process Control 15 (2005) 559-572.

[17] X. Lu, Y.-S. Yang, Q.-G. Wang, W.-X. Zheng, A double two-degree-offreedom control scheme for improved control of unstable delay processes, Journal of Process Control 15 (2005) 605-614.

[18] P. García, P. Albertos, T. Hägglund, Control of unstable non-minimumphase delayed systems, Journal of Process Control 16 (10) (2006) 10991111.

[19] J. E. Normey-Rico, E. F. Camacho, Unified approach for robust dead-time compensator design, Journal of Process Control 19 (1) (2009) 38-47.

[20] W. Tan, Analysis and design of a double two-degree-of-freedom control scheme, ISA Transactions 49 (3) (2010) 311-317.

[21] K. G. Begum, A. S. Rao, T. Radhakrishnan, Enhanced IMC based PID controller design for non-minimum phase (NMP) integrating processes with time delays, ISA Transactions, available online 18 March 2017.

[22] H.-Q. Zhou, Q.-G. Wang, L. Min, Modified smith predictor design for periodic disturbance rejection, ISA Transactions 46 (4) (2007) 493-503.

[23] D. Zheng, J. Fang, Z. Ren, Modified smith predictor for frequency identification and disturbance rejection of single sinusoidal signal, ISA Transactions 49 (1) (2010) 95-105

[24] M.-H. Tsai, P.-C. Tung, A robust disturbance reduction scheme for linear small delay systems with disturbances of unknown frequencies, ISA Transactions 51 (3) (2012) 362-372.

[25] Z. Palmor, Time-delay compensation: Smith Predictor and its modifications, The control handbook 1 (1996) 224-229.

[26] T. L. Santos, Modified artstein predictor for lti systems with dead time and unkown disturbances, Journal of Control, Automation and Electrical Systems 27 (3) (2016) 263-273.

[27] T. L. Santos, B. P. Silva, L. Uzêda, Multivariable filtered smith predictor for systems with sinusoidal disturbances, International Journal of Systems Science 48 (10) (2017) 2182-2194.

[28] P. García, P. Albertos, Robust tuning of a generalized predictor-based controller for integrating and unstable systems with long time-delay, Journal of Process Control 23 (8) (2013) 1205-1216.

[29] W. Michiels, S. I. Niculescu, On the delay sensitivity of smith predictors, International journal of systems science 34 (8-9) (2003) 543-551.

[30] J. Normey-Rico, E. Camacho, Control of dead-time processes, Springer Science \& Business Media, 2007.

[31] R. H. Middleton, D. E. Miller, On the achievable delay margin using LTI control for unstable plants, IEEE Transactions on Automatic Control 52 (7) (2007) 1194-1207.

[32] S. Mondié, W. Michiels, Finite spectrum assignment of unstable timedelay systems with a safe implementation, IEEE Transactions on Automatic Control 48 (12) (2003) 2207-2212.

[33] Q.-C. Zhong, G. Weiss, A unified smith predictor based on the spectral decomposition of the plant, International Journal of Control 77 (15) (2004) 1362-1371

[34] Q.-C. Zhong, On distributed delay in linear control laws-part I: discretedelay implementations, IEEE Transactions on Automatic Control 49 (11) (2004) 2074-2080. 